\title{
Optimizing Muscle Wrap Orientation for Aortomyoplasty
}

\author{
Brian L. Cmolik, Dirk R. Thompson, J. Timothy Sherwood, Aleksandr L. Rovner,
} Alexander S. Geha and David T. George

\author{
Division of Cardiothoracic Surgery, Case Western Reserve University School of \\ Medicine, Cleveland, Ohio, USA
}

\begin{abstract}
Background: Aortomyoplasty is a treatment for heart failure in which the latissimus dorsi muscle is wrapped around the aorta and stimulated to contract during diastole to provide chronic diastolic counterpulsation. We hypothesized that the manner in which the latissimus dorsi muscle is wrapped around the aorta determines the effectiveness of counterpulsation.

Methods: Nine mongrel dogs were studied. The left latissimus dorsi muscle (LDM) was isolated and wrapped around the descending thoracic aorta using three different muscle wrap techniques- a helical coil (HC) wrap, a circumferential (CM) wrap, and a "wringer" (WR) wrap. The three wraps were done in each dog. Left-ventricular and aortic pressures and coronary and aortic blood flows proximal and distal to the muscle-wrapped region were measured. Mean diastolic aortic pressure, endocardial-viability ratio, coronary blood flow, and blood volume ejected from the wrapped segment (wrap stroke volume) were calculated. Results: Wrap stroke volume was greater for the WR $(5.24 \pm 1.00 \mathrm{~mL}, \mathrm{p}<0.05$ compared with HC), compared with the CM $(3.70 \pm 0.64 \mathrm{~mL})$ or the $\mathrm{HC}(1.96 \pm 0.83 \mathrm{~mL})$. The increases in mean diastolic aortic pressure were similar for the WR $(10.6 \pm 4.7 \%)$ and $\mathrm{CM}$ $(9.7 \pm 4.4 \%)$ wraps and these were greater than the HC wrap (4.9 $\pm 4.7 \%)$. Endocardialviability ratio was increased similarly by the WR $(19.3 \pm 10.8 \%)$ and CM $(19.8 \pm 14.6 \%)$ wraps and these increases were greater than observed during the HC wrap $(10.5 \pm 6.3 \%)$. The WR wrap provided the greatest increase in coronary blood flow $(24.3 \pm 23.0 \%)$ compared with the CM $(17.8 \pm 12.9 \%)$ and $\mathrm{HC}(5.7 \pm 4.6 \%)$.

Conclusion: These data suggest that: 1) all muscle wrap techniques effect diastolic counterpulsation; 2) muscle wrap orientation influences ameliorative potential; and 3) wringer and adjacent are better than helical coil. Chronic studies should be done to test whether the differences are maintained.
\end{abstract}

Key words : aortomyoplasty, diastolic counterpulsation, skeletal muscle cardiac assist.

Basic Appl. Myol. 9 (4): 167-173, 1999

\begin{abstract}
Aortomyoplasty is a new form of cardiac assistance whereby autologous skeletal muscle is wrapped around the ascending [7]or descending [2-6, 10, 15, 20-23, 25, 30 thoracic aorta and electrically stimulated to contract during diastole. The underlying mechanisms by which aortomyoplasty can assist a failing heart are analogous to the intra-aortic balloon pump (IABP). Whereas the IABP must be removed in a matter of days or weeks, aortomyoplasty offers means to effect chronic diastolic counterpulsation. The primary effects of diastolic counterpulsation (using either IABP or aortomyoplasty) are increasing myocardial oxygen supply (by increasing mean diastolic aortic pressure), and reducing myocar-
\end{abstract}

dial oxygen consumption (via afterload reduction). Until recently, aortomyoplasty has been limited to research applications; however, clinical experiences using the latissimus dorsi muscle (LDM) have been reported in 28 patients [6,7].

The effects of intra-aortic balloon volume and placement have been extensively investigated, but the effects of various muscle wrap techniques have not been compared. Just as balloon size and position influence the effectiveness of the IABP, the technique used to wrap skeletal muscle around the aorta may influence the effectiveness of aortomyoplasty. Factors influencing the potential of a particular wrap technique include the 
length of the wrapped aortic segment, the diameter of the aorta, the fiber-orientation of the muscle, and the portion of the muscle with the best blood supply. An ideal wrap technique would balance the factors affecting diastolic counterpulsation to maximize the ameliorative potential of aortomyoplasty.

Two techniques for wrapping the descending thoracic aorta are reported in the literature. In one technique, the latissimus dorsi muscle (LDM) is wrapped en bloc around the aorta and secured to itself $[3-5,10,13,23$, $25,30]$. In the second technique, a $4-5 \mathrm{~cm}$ wide strip of the lateral portion of LDM is isolated and wrapped as a helical coil around the descending thoracic aorta $[2,14$, $20,21]$. These two wrap techniques have not been compared using a consistent experimental protocol.

In this study, we compared three descending thoracic aortomyoplasty techniques - the two previously eported wrap techniques and a new technique designed to maximize the cardiovascular effects of counterpulsation. In mongrel dogs, we performed acute aortomyoplasty and recorded indices of diastolic counterpulsation to compare the effectiveness of the three wrap techniques. Results suggest wrap technique can significantly influence the effectiveness of diastolic counterpulsation, and that the new wrap technique proved most effective among the three wraps tested.

\section{Methods}

In mongrel dogs $(n=12,20-25 \mathrm{~kg})$, three muscle wrap techniques were investigated. The animals received humane care as outlined in the Principles of Laboratory Animal Care formulated by the National Society for Medical Research and the Guide for the Care and Use of Laboratory Animals prepared by the National Academy of Sciences and published by the National Institute of Health (NIH publication 85-23, revised 1985).

Each dog was anesthetized with pentobarbital sodium (25 mg/kg) and intubated. Limb-lead ECG was monitored. The left LDM was isolated leaving the neurovascular pedicle intact. Pacing electrodes (TPW20, Ethicon, Somerville, NJ) were positioned intramuscularly in the region of the thoracodorsal nerve. The muscle was divided longitudinally along the anatomical boundaries of the lateral and oblique portions $[8,28]$. A left thoracotomy was performed at the fifth intercostal space. The muscle was transposed into the chest through the bed of the third rib. The intercostal arteries of the descending thoracic aorta were ligated so LDM could be wrapped around the proximal descending thoracic aorta.

Solid state pressure transducers (MPC-500, Millar, Inc. Houston, TX) were positioned in the left ventricle (through the apex), and in the ascending aorta (via the left carotid artery), to measure left ventricular (LVP) and aortic pressures (AP), respectively (Figure 1). The left anterior descending (LAD) coronary artery and the

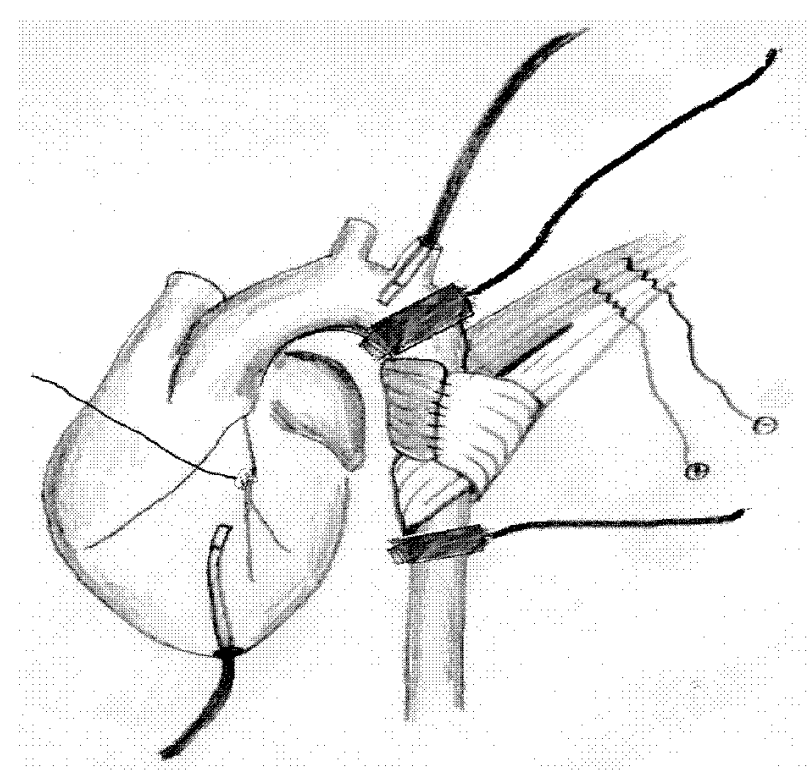

Figure 1. Full instrumentation study.

aorta distal to the muscle wrap were instrumented with Doppler ultrasonic flow probes (Transonic, Inc., Ithaca, NY). In eight dogs, an additional aortic flow probe was positioned on the aorta proximal to the muscle wrap (immediately distal to the left subclavian artery). For these eight subjects, flow probes where thus positioned both proximal and distal to the wrapped aortic segment - facilitating calculation of the blood volume ejected from the wrapped aortic segment.

The three wrap configurations investigated in this study were termed 1) helical coil, 2) wringer, and 3) circumferential (Figure 2). The helical coil consisted of the lateral portion of the split LDM wrapped clockwise around the descending aorta — covering the aorta from the subclavian artery for approximately $8 \mathrm{~cm}$ (Figure 2a). The wringer wrap consisted of the obliquetransverse portion of the LDM wrapped clockwise around the superior portion of the descending thoracic aorta, coupled to the lateral portion of LDM, which was wrapped in a counterclockwise direction distal to the oblique-transverse portion (Figure $2 b$ ). The circumferential wrap used the entire width of LDM (both the lateral and oblique-transverse portions adjacent to one another) wrapped clockwise around the aorta (Figure 2c). The three wraps were evaluated in a rotating order so that each wrap was done first, second, or third, on each
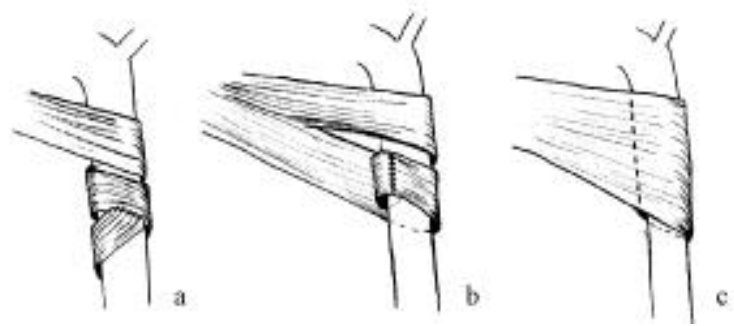

Figure 2. Three muscle wrap types: a) helical coil; b) wringer; c) circumferential. 


\section{Aortomyoplasty wrap geometry}

dog, with an equal number of times among the 12 dogs.

For each wrap, the LDM was stimulated with a pulse train to initiate contraction at the dicrotic notch. Stimu lus train duration was set based on heart rate, such that the muscle relaxed just prior to the opening of the aortic valve in the ensuing systole. Intra-train stimulus frequency was $50 \mathrm{~Hz}$ and pulse width was $210 \mu \mathrm{s}$.

Muscles were stimulated in alternate diastoles (1:2 mode) for this investigation. A physiologic recorder (Gould Inc. Cleveland, $\mathrm{OH}$ ) detected the R-wave, and a computer-controlled stimulator developed in our laboratory [8] triggered a second, electrically-isolated stimulator (S8800/SIU5, Grass Instruments, Quincy, MA) in 1:2 mode.

Data were digitally sampled (AT-MIO-64E, National Instruments, Austin, TX) at $500 \mathrm{~Hz}$ and stored to hard disk.

\section{Data analysis}

Stimulated and unstimulated beats were identified and analyzed for each muscle wrap. Mean diastolic aortic pressure (MDAP) was calculated as the mean aortic pressure during the time when aortic pressure exceeded left-ventricular pressure. Coronary minute-flow in the left-anterior descending coronary artery was calculated by multiplying the integral of coronary flow (over individual cardiac cycles) by the heart rate. Endocardial viability ratio (EVR) was calculated as the diastolic pressure-time integral (DPTI) divided by the left-ventricular tension-time index (TTI). The difference between proximal \& distal aortic flows, the differential flow, demonstrates total wrap ejection (during diastole) and filling (during systole). The wrap stroke volume (WSV) was calculated from the integral of differential flow. Wrap output (WO) was then calculated as the WSV multiplied by the number of muscle contractions per minute (note that in 1:2 mode the number of muscle contractions per minute equalled half of the heart rate). Peak left-ventricular pressure (PLVP), peak $+\mathrm{dP} / \mathrm{dt}$, and peak $-\mathrm{dP} / \mathrm{dt}$ were calculated from measurements of leftventricular pressure. Left-ventricular end-diastolic pressure (EDP) was defined as the left-ventricular pressure when $\mathrm{dP} / \mathrm{dt}$ equalled 10 percent of peak $+\mathrm{dP} / \mathrm{dt}$. The ventricular relaxation constant $(\tau)$, was calculated via an exponential fit of left-ventricular pressure as a function of time (peak - dP/dt to $5 \mathrm{~mm} \mathrm{Hg}$ above EDP).

Data are presented as mean \pm standard error of the mean for stimulated and unstimulated cycles. Repeated measures analysis of variance (ANOVA) was used to find significant differences between stimulated and unstimulated cycles, and wrap types. When significant differences were found $p<0.05$ ), a Student-NewmanKeuls test was used for multiple comparisons.

\section{Results}

Sample data (recorded during aortic counterpulsation in 1:2 mode) are shown in Figure 3. The top panel shows left-ventricular and aortic pressures, together with the ECG (the closely spaced spikes on the ECG are from the electrical pulses stimulating the assisting mu scle). In the sample data, MDAP increased by about $10 \mathrm{~mm} \mathrm{Hg}$ during muscle stimulation and peak leftventricular pressure (PLVP) was reduced in the cardiac systole following muscle contraction. The middle panel shows aortic blood flow proximal and distal to the mu scle wrapped region. During muscle stimulation, proximal flow became more negative (flow was directed cephalad) and distal flow became more positive (flow was directed caudad), when compared to unstimulated cycles. The bottom panel shows the difference between the proximal and distal aortic flows. In this differential flow trace, positive flow represents wrap ejection and negative flow represents wrap filling. During muscle contraction, there was a net outflow from the wrapped segment; during muscle relaxation, there was a net inflow.

In general, each wrap technique successfully produced diastolic counterpulsation. Stimulation of either the wringer or circumferential wraps effected similar results (with the wringer slightly superior while the helical coil effected more modest results (Table 1). Compared to baseline cardiac cycles, muscle stimulation resulted in significant increases in MDAP and EVR - regardless of wrap technique. Muscle stimulation increased MDAP from $93.1 \pm 4.2$ to $96.9 \pm 4.4 \mathrm{~mm} \mathrm{Hg}$ using the helical coil wrap, from $92.1 \pm 4.6$ to $99.9 \pm 4.8 \mathrm{~mm} \mathrm{Hg}$ using the
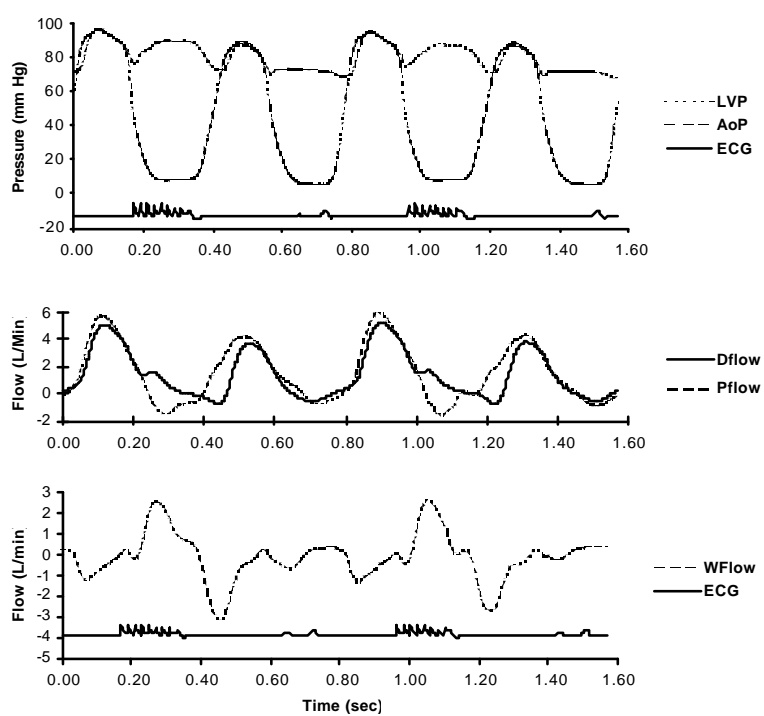

Figure 3. Sample hemodynamic data with aortomyoplasty in 1:2 counterpulsation mode. Top panel: Left-ventricular (LVP) and aortic pressures (AoP) with electrocardiogram (ECG). Middle panel: Aortic blood flow proximal (Pflow) and distal (Dflow) to the muscle wrapped region. Bottom panel: Difference between proximal and distal aortic flows. Positive flow represents wrap filling and negative flow represents wrap filling. 


\section{Aortomyoplasty wrap geometry}

Table 1. Hemodynamic data for the three muscle wraps. Muscle was stimulated during diastole in a 1:2 muscle-to-cardiac ratio. Stimulated beats are compared to unstimulated baseline beats for each wrap. MDAP = mean diastolic aortic pressure; $C Q=$ coronary blood flow; $E V R=$ endocardial viability ratio; $W S V=$ wrap stroke volume; $W O=w r a p$ blood output; $P L V P=$ peak left-ventricular pressure; $E D P=$ end-diastolic pressure $;+d P / d t=$ maximum positive change in ventricular pressure; $-d P / d t=$ maximum negative change in ventricular pressure; $T a u(\tau)=$ constant of isovolumic pressure relaxation, was calculated as the negative reciprocal of the slope of the plot of the natural logarithm of left ventricular pressure versus time from maximum negative dP/dt (maximum rate of decrease of left ventricular pressure) to $5 \mathrm{~mm} \mathrm{Hg}$ above the left ventricular end-diastolic pressure. Data were compared using repeated measures ANOVA. $* p<0.05$ compared to baseline $; \dagger p<0.05$ compared to helical coil wrap; $\neq$ compared to circumferential wrap.

\begin{tabular}{|c|c|c|c|c|c|c|}
\hline Wrap Type & & $\begin{array}{l}\text { MDAP } \\
(\mathrm{mm} \mathrm{Hg})\end{array}$ & $\begin{array}{c}\mathrm{CQ} \\
(\mathrm{ml} / \mathrm{min})\end{array}$ & EVR & $\begin{array}{r}\text { WSV } \\
(\mathrm{ml})\end{array}$ & $\begin{array}{c}\text { WO } \\
(\mathrm{ml} / \mathrm{min}, 1: 2)\end{array}$ \\
\hline \multirow[t]{2}{*}{ helical coil } & baseline & $93.1 \pm 4.2$ & $12.1 \pm 1.2$ & $1.27 \pm 0.06$ & - & - \\
\hline & stimulated & $96.9 \pm 4.4^{*}$ & $12.7 \pm 1.3$ & $1.37 \pm 0.06^{*}$ & $1.5 \pm 0.4$ & $101 \pm 31$ \\
\hline \multirow[t]{2}{*}{ wringer } & baseline & $92.1 \pm 4.6$ & $12.2 \pm 1.7$ & $1.28 \pm 0.06$ & - & - \\
\hline & stimulated & $99.9 \pm 4.8^{*}$ & $14.1 \pm 1.9^{*}$ & $1.48 \pm 0.07 *+\neq$ & $3.3 \pm 0.8 \dagger$ & $179 \pm 63 \dagger$ \\
\hline \multirow[t]{2}{*}{ circumferential } & baseline & $94 \pm 3.7$ & $12.2 \pm 1.4$ & $1.21 \pm 0.06$ & - & - \\
\hline & stimulated & $100.9 \pm 3.6^{*}$ & $13.6 \pm 1.5^{*}$ & $1.38 \pm 0.07^{*}$ & $2.9 \pm 0.7 \dagger$ & $153 \pm 54 \dagger$ \\
\hline \multicolumn{2}{|l|}{ Wrap Type } & $\begin{array}{c}\text { PLVP } \\
(\mathrm{mm} \mathrm{Hg})\end{array}$ & $\begin{array}{c}\mathrm{EDP} \\
(\mathrm{mm} \mathrm{Hg})\end{array}$ & $\begin{array}{c}+\mathrm{dP} / \mathrm{dt} \\
(\mathrm{mm} \mathrm{Hg} / \mathrm{s})\end{array}$ & $\begin{array}{c}-\mathrm{dP} / \mathrm{dt} \\
(\mathrm{mm} \mathrm{Hg} / \mathrm{s})\end{array}$ & $\begin{array}{l}\operatorname{tau} \\
(\mathrm{ms})\end{array}$ \\
\hline \multirow[t]{2}{*}{ helical coil } & baseline & $107.5 \pm 4.5$ & $7.4 \pm 1$ & $2263 \pm 215$ & $-2889 \pm 281$ & $35.5 \pm 2.8$ \\
\hline & stimulated & $106.6 \pm 4.4$ & $6.5 \pm 1 *$ & $2081 \pm 192 *$ & $-2835 \pm 272$ & $35.4 \pm 2.8$ \\
\hline \multirow[t]{2}{*}{ wringer } & baseline & $107.9 \pm 4.7$ & $6.8 \pm 0.7$ & $2067 \pm 137$ & $-2805 \pm 260$ & $35.2 \pm 3.2$ \\
\hline & stimulated & $107.7 \pm 4.5$ & $6.2 \pm 0.7 *$ & $1929 \pm 142$ & $-2813 \pm 270$ & $35.8 \pm 3.4$ \\
\hline \multirow[t]{2}{*}{ circumferential } & baseline & $110.0 \pm 3.7$ & $8.5 \pm 1$ & $2120 \pm 161$ & $-2942 \pm 223$ & $36.6 \pm 2.9$ \\
\hline & stimulated & $108.8 \pm 3.9$ & $7.6 \pm 1.1^{*}$ & $1961 \pm 137 *$ & $-2853 \pm 207$ & $36.3 \pm 2.9$ \\
\hline
\end{tabular}

wringer wrap, and from $94 \pm 3.7$ to $100.9 \pm 3.6 \mathrm{~mm} \mathrm{Hg}$ using the circumferential wrap. Stimulation increased EVR from $1.27 \pm 0.06$ to $1.37 \pm 0.06$ using the helical coil wrap, from $1.28 \pm 0.06$ to $1.48 \pm 0.07$ using the wringer wrap, and from $1.21 \pm 0.06$ to $1.38 \pm 0.07$ using the circumferential wrap. The circumferential wrap effected a significantly greater EVR than the helical coil, and the wringer wrap effected a significantly greater EVR than either the helical coil or circumferential wrap.

Counterpulsation increased coronary minute-flow (CQ) from $12.1 \pm 1.2$ to $12.7 \pm 1.3 \mathrm{ml} / \mathrm{min}$ using the helical coil wrap, from $12.2 \pm 1.7$ to $14.1 \pm 1.9 \mathrm{ml} / \mathrm{min}$ using the wringer wrap, and from $12.2 \pm 1.4$ to $13.6 \pm 1.5$ $\mathrm{ml} / \mathrm{min}$ using the circumferential wrap. Compared to baseline, the increase in CQ using either the wringer or circumferential wrap was statistically significant.

The helical coil wrap ejected the least volume (WSV), the wringer ejected the greatest volume, and the circumferential wrap ejected an intermediate volume. With 1:2 mode stimulation, the wrap output (WO) for the helical coil, wringer, and circumferential wraps were $101 \pm 31$, $179 \pm 63$, and $153 \pm 54 \mathrm{ml} / \mathrm{min}$, respectively. The volume ejected by the wringer or circumferential wrap was significantly greater than the volume ejected by the helical coil wrap.
Compared to baseline, EDP was significantly lower following muscle stimulation - from $7.4 \pm 1.0$ to $6.5 \pm 1.0 \mathrm{~mm} \mathrm{Hg}$ following stimulation of the helical coil, from $6.8 \pm 0.7$ to $6.2 \pm 0.7 \mathrm{~mm} \mathrm{Hg}$ following stimulation of the wringer wrap, and from $8.5 \pm 1.0$ to $7.6 \pm 1.1$ $\mathrm{mm} \mathrm{Hg}$ following stimulation of the adjacent wrap. The PLVP was reduced slightly by counterpulsation - with no difference among wrap techniques. The $+\mathrm{dP} / \mathrm{dt}$ was significantly greater in all three wraps when compared to baseline. However, there were no differences among the three wraps. The $-\mathrm{dP} / \mathrm{dt}$ and $\tau$ were unaffected by aortic counterpulsation.

\section{Discussion}

The success of counterpulsation by aortomyoplasty is related to how effectively muscle contraction decreases aortic cross sectional area and ejects blood from the wrapped aortic segment. The amount of blood ejected from the wrapped aortic segment is influenced by both aortic and muscle factors. Factors relating to the aorta include the inside and outside vessel diameter, the length of the wrapped aortic segment, and aortic compliance. Factors relating to the muscle include the force and speed of contraction, as well as muscle powergenerating ability. These muscle factors are influenced by the anatomic region of the muscle flap being used. In 


\section{Aortomyoplasty wrap geometry}

turn, all of these aortic and muscle variables are interdependent, based on the geometric relationship between the linearly contracting skeletal muscle and the cylindrical aorta.

A large aortic diameter together with greatest possible muscle-to-aorta coverage will maximize the effects of muscle contraction during aortomyoplasty. A method of maximizing the volume of the wrapped aortic segment involves placing a pericardial patch to enlarge aortic diameter $[4,5]$. Aortic compliance varies minimally from subject to subject, though decreases in compliance are seen in the diseased aorta. The speed, force, and powergenerating ability of the muscle are known to diminish following conversion of the muscle to fatigue resistance [24], though researchers are considering methods of manipulating skeletal muscle adaptation [1, 12, 16]or improving its performance through advanced methods of activation [19, 26, 27, 29]. Furthermore, it is unclear whether diminished muscle force following chronic electrical stimulation decreases the effectiveness of aortomyoplasty [9].

This study varied muscle wrap geometry as a means to optimize the hemodynamic benefits of aortomyoplasty. To displace blood from the wrapped aortic segment, the force of a linearly contracting muscle must be translated into a decrease in volume of the cylindrical aorta. Since volume changes in a cylinder are related to the square of the changes in radius, it is more efficient to decrease the volume of a cylinder by decreasing its cross-sectional area (as opposed to decreasing its length). A wrap geometry that maximizes the circumferential component of the muscle force vector should provide the greatest decrease in aortic volume. In the helical coil, muscle fibers are oriented at an angle. This could be one reason why the helical coil wrap was not as efficient in effecting counterpulsation. The circumferential and wringer wraps have force vectors more closely aligned with the short axis of the aorta.

Regional perfusion of the assisting LDM also influences the efficacy of counterpulsation via aortomy oplasty. During isolation, the distal region of LDM suffers ischemia and contractile dysfunction [11, 1618]Following a vascular delay, the muscle partially recovers [16-18]. Since the helical coil wrap makes use of the distal muscle region to cover the aorta, a potential reason for the ineffectiveness of the helical coil wrap in this study is distal muscle ischemia. The circumferential and wringer wraps do not rely as much on the distal ischemic region.

The proximal portion of healthy and well-perfused muscle not directly wrapped around the aorta might also affect wrap function. For the helical coil and circumferential muscle wraps, the well-perfused proximal portions cannot contribute to wrap ejection because contraction of the proximal muscle region displaces the aorta laterally but does not decrease the aortic cross sectional area. For the wringer wrap, the muscle wrapped clockwise and counterclockwise fashion forms a loop around the aorta — pulling the ends of this loop produces a garrote-like effect to augment ejection.

Two muscle wrap techniques have been reported in aortomyoplasty of the descending thoracic aorta helical coil and adjacent. Successful counterpulsation using each muscle wrap has been reported following chronic muscle stimulation, however, a comparison of these two wrap techniques has not been made.

The potential advantages of aortomyoplasty as a circulatory support technique include the use of the most proximal muscle portion for the basis of the wrap. The thoracodorsal artery is the basis for the blood supply to this muscular flap. The more proximal muscle used, the less likelihood of distal muscle fibrosis effecting the performance of the wrap. The procedure can be performed entirely through a left thoracotomy without need for additional incisions. Muscle training may begin within days of the procedure, as opposed to weeks for cardiomyoplasty, thus enabling the procedure to give the patient early benefit during training. Finally, there will be no early impairment of cardiac function because the muscle is wrapped around the aorta as opposed to around the heart. Contraindications to aortomyoplasty are severely calcified or atherosclerotic aorta, aortic valvular insufficiency, or severe heart failure (NYHA Class IV), in which a more aggressive treatment (such as the left-ventricular assist device) is required.

We have shown that muscle wrap geometry influences counterpulsation with aortomyoplasty. A critical factor in determining wrap performance is the volume of blood a given wrap can eject. Compared to the two wrap techniques used clinically (circumferential and helical coil) the new wringer technique provides modest advantages. Additionally, the helical coil wrap proved the poorest, and should not be chosen over the circumferential or wringer. This information should be considered when applying aortomyoplasty to patients.

This study is limited because it utilized an acutely isolated, unconditioned skeletal muscle. The acutely isolated muscle suffers greater ischemia because it has not had the vascular delay period. Lack of a vascular delay may have had greater impact on the helical coil wrap, which uses more of the distal muscle region than the circumferential and wringer wraps. This acute study may have been further limited because the muscle was not adhered to the aorta (as will occur chronically). Since there were no adhesions between the aorta and LDM in this acute study, afterload reduction - partly attributable to the opening of the aortic lumen by elastic recoil of the adhered skeletal muscle - was likely reduced. Since the LDM was not conditioned for this study, it had greater strength and speed than a chroni- 


\section{Aortomyoplasty wrap geometry}

cally stimulated muscle. However, preliminary results from our laboratory suggest that muscle conditioning may have only minor affects on aortomyoplasty [9]. We believe that factors such as distal muscle ischemia and muscle-to-aortic adhesions are more dominant than the absolute strength of the LDM. An important question for future work is whether the wringer wrap, which equires splitting of the LDM has any deleterious effects on the muscle. Acute pilot studies accomplished by our laboratory (unpublished), in which the contractile performance of the canine LDM was measured before and after dividing the muscle for the wringer wrap, suggest there is no diminution in muscle performance following the division.

\section{Acknowledgement}

This work was supported in part by a Research Initiation Grant from the Ohio Board of Regents Challenge Fund, and the American Heart Association, Northeast Ohio Affiliate.

\section{Address correspondence to:}

Brian L. Cmolik, MD, Division of Cardiothoracic Surgery, Case Western Reserve University School of Medicine, 11100 Euclid Avenue, Cleveland, Ohio, USA, 44106-5011, phone 216844 3055, fax 216844 7597, Email blc3@po.cwru.edu.

\section{References}

[1] Arpesella G, Mikus PM, Lombardi P, Pierangeli A, Giannoni A, Zampieri S, Catani C, Carraro U: Activity-rest regimen of latissimus dorsi stimulation for cardiomyoplasty: Anatomy, isomyosin and sustained power of sheep LD up to one year. Basic and Applied Myology 1997; 7: 45-53.

[2] Cardone JC, Yoon PD, Trumble DR, Magovern JA: Regional effects of aortomyoplasty in acute ischemia. Ann Thorac Surg 1996; 61: 426-9.

[3] Cernaianu AC, Vassillidize TV, Flum DR, Gallucci JG, Olah A, Cilley JH, Jr., Gorsso MA, DelRossi AJ: Latissimus dorsi and serratus anterior dynamic descending aortomyoplasty for ischemic cardiac failure. Ann Thorac Surg 1995; 59: 639-43.

[4] Chachques JC, Grandjean PA, Cabrera Fischer EI, Latremouille C, Jebara VA, Bourgeois I, Carpentier A: Dynamic aortomyoplasty to assist left ventricular failure. Ann Thorac Surg 1990; 49: 225-30.

[5] Chachques JC, Haab F, Cron C, Fischer EC, Grandjean P, Bruneval P, Acar C, Jebara VA, Fontaliran F, Carpentier AF: Long-term effects of dynamic aortomyoplasty. Ann Thorac Surg 1994; 58: 12834.

[6] Chachques JC, Radermercker M, Grandjean PA, Chaussende F, Carpentier AF: Dynamic aortomy oplasty for long-term circulatory support: experi- mental studies and clinical experience, in Carpentier AF, Chachques JC, Grandjean PA (eds): Cardiac Bioassist. Armonk, NY, Futura Publishing Co., Inc., 1997, pp 481-9.

[7] Chachques JC, Radermercker M, Tolan MJ, Fischer EI, Grandjean PA, Carpentier AF: Aortomyoplasty counterpulsation: experimental results and early clinical experience. Ann Thorac Surg 1996; 61: 420-5.

[8] Cheever EA, Birge JR, Thompson DR, Santamore WP, George DT: A versatile microprocessor-based multi-channel muscle stimulator for skeletal muscle cardiac assist. IEEE Transactions on Biomedical Engineering 1998; 45: 56-67.

[9] Cmolik BL, Thompson DR, Cheever EA, Geha AS, George DT: Beneficial effects of aortomyoplasty are maintained following chronic muscle conditioning. Journal of Heart Failure 1997; 4: 187.

[10] Constance CG, Sabini G, Turi GK, Hines GL: Descending thoracic aortomyoplasty: effect of chronically conditioned muscle on heart failure. Cardiovasc Surg 1993; 1: 291-5.

[11] Cruz MP, Michele JJ, Mannion JD, Magno M, George DT, Santamore WP: Cardiomyoplasty. Latissimus dorsi muscle function and blood flow during isolation. ASAIO Journal 1997; 43: 338-44.

[12] Ferguson AS, Stone HE, Roessmann U, Burke M, Tisdale E, Mortimer JT: Muscle plasticity: comp arison of a $30-\mathrm{Hz}$ burst with $10-\mathrm{Hz}$ continuous stimulation. J Appl Phys 1989; 66: 1143-51.

[13] Fischer EI, Chachques JC, Christen AI, Risk MR, Carpentier AF: Benefits of aortic and pulmonary counterpulsation using dynamic latissimus dorsi myoplasty. Ann Thorac Surg 1995; 60: 417-21.

[14] Hakami A, Santamore WP, Stremel RW, Pedersen EM: Evaluation of stimulation parameters on aortomyoplasty, using latissimus dorsi muscle in a goat model (an acute study). European Surgical Research 1995; 27: 136

[15] Hines GL, Mishriki Y, Williams L, Monroe K, Metwally N: Physiologic and pathologic evaluation of chronic extra-aortic counterpulsation with latissimus dorsi flap. Preliminary results. J Cardiovasc Surg (Torino) 1991; 32: 485-90.

[16] Ianuzzo CD, Ianuzzo SE，Anderson WA: Cardiomyoplasty: transformation of the assisting muscle using intermittent versus continuous stimulation. J Card Surg 1996; 11: 293-303.

[17] Ianuzzo CD, Ianuzzo SE, Carson N, Feild M, Locke M, Gu J, Anderson WA, Klabunde RE: Cardiomyoplasty: degeneration of the assisting skeletal muscle. J Appl Phys 1996; 80: 1205-13. 


\section{Aortomyoplasty wrap geometry}

[18] Kratz JM, Johnson WS, Mukherjee R, Hu J, Crawford FA, Spinale FG: The relation between latissimus dorsi skeletal muscle structure and contractile function after cardiomyoplasty. J Thorac Cardiovasc Surg 1994; 107: 868-78.

[19] Kwende MM, Jarvis JC, Salmons S: The inputoutput relations of skeletal muscle. Proceedings of the Royal Society of London - Series B: Biological Sciences 1995; 261: 193-201.

[20] Lazzara RR, Trumble DR, Magovern JA: Autogenous cardiac assist with chronic descending thoracic aortomyoplasty. AnnThorac Surg 1994; 57: 1540-4.

[21] Lazzara RR, Trumble DR, Magovern JA: Chronic counterpulsation with descending thoracic aortomyoplasty improved cardiac function in animals with heart failure. Journal of Heart and Lung Transplantation 1994; 13: 652-60.

[22] Lazzara RR, Trumble DR, Magovern JA: Dynamic descending thoracic aortomyoplasty: comparison with intraaortic balloon pump in a model of heart failure. Ann Thorac Surg 1994; 58: 366-70.

[23] Pattison CW, Cumming DV, Williamson A, Clayton-Jones DG, Dunn MJ, Goldspink G, Yacoub M: Aortic counterpulsation for up to 28 days with autologous latissimus dorsi in sheep. $J$ Thorac Cardiovasc Surg 1991; 102: 766-73.

[24] Salmons S, Jarvis JC: The working capacity of skeletal muscle transformed for use in a cardiac assist role, in Chiu RCJ, Bourgeois IM (eds): Transformed Muscle for Cardiac Assist and Repair.
Mount Cisco, NY, Futura Publishing Company, 1990, pp 89-104.

[25] Takahashi R, Yozu R, Kurosaka Y, Kawada S: Assisted circulation using cardiomyoplasty together with aortomyoplasty. Artificial Organs 1993; 17: 914-8.

[26] Thoma H, Frey M, Girsch W, Gruber H, Happak W, Lanmuller H, Losert U, Mayr W: First experimental application of multichannel stimulation devices for cardiomyoplasty. Journal of Cardiac Surgery 1991; 6: 252-8.

[27] Thompson DR, Michele JJ, Cheever EA, George DT: Regional electrical activation of the latissimus dorsi muscle for cardiac assist, The Cardiac Bioelectricity Research and Training Center (CBRTC) Retreat, Cleveland, OH, 1996. Case Western Reserve School of Engineering and School of Medicine.

[28] Thompson DR, Michele JJ, Cheever EA, George DT: Selective stimulation of skeletal muscle for cardiac assist. Annals of Biomedical Engineering 1995; 22: 196.

[29] Thomsen M, Veltink PH: Influence of synchronous and sequential stimulation on muscle fatigue. $\mathrm{Me}$ dical and Biological Engineering and Computing 1997; 35: 186-92.

[30] Vertrees RA, Cernaianu AC, Camishion RC, Cilley J, Jr., Baldino WA, DelRossi AJ: A surgical approach to chronic aortomyoplasty in the goat model. Journal of Investigative Surgery 1993; 6: 419-29. 